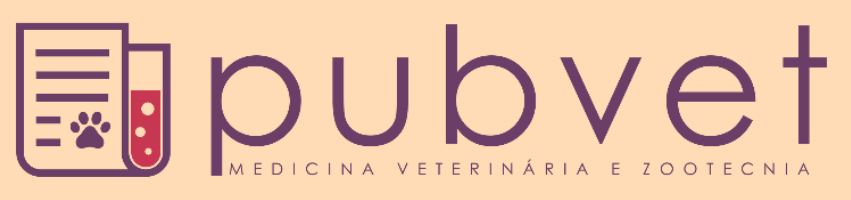

https://doi.org/10.31533/pubvet.v15n07a861.1-7

\title{
Percepção da população sobre doença renal crônica em animais de companhia em Manaus, Amazonas
}

\author{
Matheus Henrique Nogueira ${ }^{1} \bullet$, Marina Pandolphi Brolio ${ }^{2 *} \bullet$

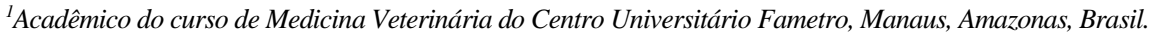 \\ ${ }^{2}$ Coordenadora do curso de Medicina Veterinária do Centro Universitário Fametro, Manaus, Amazonas, Brasil. \\ *Autor para correspondência, E-mail: marina.brolio@fametro.edu.br
}

\begin{abstract}
Resumo. Animais de companhia estão cada vez mais presentes nos lares brasileiros, assumiram importante papel na relação intrafamiliar e geram maior interesse dos tutores com sua saúde. Contudo, há poucos dados disponíveis sobre o grau de conhecimento dos tutores sobre sanidade e bem-estar animal de cães e gatos no que se refere a enfermidades relevantes, como a Doença Renal Crônica (DRC), doença silenciosa em estágios iniciais, de difícil controle e frequentemente diagnosticada apenas em fases avançadas. O objetivo deste trabalho foi avaliar o conhecimento sobre a DRC em cães e gatos entre moradores de Manaus, Amazonas e também traçar um panorama sobre os hábitos e cuidados de rotina proporcionados a esses animais. Para isso, foram entrevistadas 268 pessoas por meio de um questionário on-line através da ferramenta Google Forms. Os resultados revelaram que a maioria da população desconhece a DRC em animais e poucas pessoas levam o pet regularmente ao médico veterinário. Grande parte dos participantes procura atendimento veterinário somente em caso de doença ou vacinação animal. Um dado alarmante encontrado é que muitos entrevistados relataram nunca ter levado seu pet para uma consulta ao médico veterinário. Os resultados comprovam a importância e a necessidade de atividades de orientação e prevenção a doenças em cães e gatos a fim de manter os tutores bem informados e melhorar a qualidade de vida destes animais.
\end{abstract}

Palavras-chave: Cão, doença renal crônica, gato

\section{Perception of the population about chronic kidney disease in pets in Manaus, Amazonas}

\begin{abstract}
Companion animals are increasingly present in Brazilian homes, have taken on an important role in the intrafamily relationship, and generate greater interest of tutors on their health. However, there is little data available on the degree of tutors' knowledge about animal health and welfare of dogs and cats concerning relevant diseases, such as Chronic Kidney Disease (CKD), a silent disease in the early stages that is difficult to control and often diagnosed only in advanced stages. This work aimed to evaluate the knowledge of residents of Manaus, Amazonas on CKD in dogs and cats and provide an overview of the habits and routine care provided to these animals. To do so, we interviewed 268 people through an online questionnaire using the Google Forms platform. The results revealed that most of the population is unaware of CKD in animals, and few people take the pet regularly to the veterinarian. Most participants seek veterinary care only in case of disease or animal vaccination. An alarming finding is that many respondents reported never taking their pet for a visit to the veterinarian. The results demonstrate the importance and need for disease orientation and prevention activities in dogs and cats to keep tutors well informed and improve these animals' quality of life.
\end{abstract}

Keywords: Dog, cat, chronic kidney disease 


\title{
Percepción de la población sobre enfermedad renal crónica en animales de compañía en Manaus, Amazonas
}

\begin{abstract}
Resumen. Los animales de compañía están cada vez más presentes en los hogares brasileños, han asumido un papel importante en la relación intrafamiliar y generan un mayor interés de los tutores con su salud. Sin embargo, se dispone de pocos datos sobre el grado de conocimiento de los tutores sobre la salud animal y el bienestar de perros y gatos con respecto a enfermedades relevantes, como la Enfermedad Renal Crónica (ERC), enfermedad silenciosa en etapas tempranas, de difícil control y a menudo se diagnostica solo en etapas avanzadas. El objetivo de este trabajo fue evaluar el conocimiento sobre la ERC en perros y gatos entre los residentes de Manaus, Amazonas y también brindar una visión general de los hábitos y cuidados rutinarios que se brindan a estos animales. Para ello, se entrevistó a 268 personas a través de un cuestionario online utilizando la herramienta Google Forms. Los resultados revelaron que la mayoría de la población desconoce la ERC en los animales y pocas personas llevan a la mascota al veterinario con regularidad. La mayoría de los participantes buscan atención veterinaria solo en caso de enfermedad o vacunación animal. Un hallazgo alarmante es que muchos encuestados informaron que nunca habían llevado a su mascota a una visita al veterinario. Los resultados demuestran la importancia y la necesidad de actividades de orientación y prevención de enfermedades en perros y gatos para mantener bien informados a los tutores y mejorar la calidad de vida de estos animales.
\end{abstract}

Palabras clave: Perro, enfermedad renal crónica, gato

\section{Introdução}

A medicina veterinária evoluiu nos últimos anos, proporcionando bem-estar e maior longevidade aos animais de companhia, satisfazendo a demanda da população humana, que interage cada vez mais com esses animais (Carvalho \& Pessanha, 2013). Essa participação nos relacionamentos familiares também pode levar a uma maior preocupação com a saúde desses animais. Contudo, pesquisas são necessárias para investigar o conhecimento dos tutores em relação à saúde e necessidade dos animais de companhia, bem como sobre enfermidades importantes como a doença renal crônica (DRC).

A DRC é diagnosticada com frequência em pets (Waki et al., 2010). De maneira geral, os animais apresentam perda de peso gradativa pela falta de apetite devido às alterações gastrointestinais, poliúria e polidipsia compensatórias, incluindo episódios de êmese e diarreia esporádicos que podem levar à desidratação (Polzin, 2011). Por ser de manifestação silenciosa no estágio inicial, os tutores percebem as alterações e as identificam tardiamente, geralmente em fase avançada, quando já existe déficit funcional (Martínez \& Carvalho, 2010; Queiroz \& Fioravanti, 2014). Assim, a DRC está entre as principais causas de mortalidade em cães e gatos, especialmente os animais geriátricos devido a perdas de células renais ao longo da vida (Gerber, 2017; Togni et al., 2018).

Neste contexto, é importante investigar o conhecimento dos tutores sobre necessidades básicas de saúde e bem-estar de cães e gatos, bem como sobre as principais enfermidades que podem acomete-los. Este trabalho teve como objetivo investigar a percepção da população de Manaus/AM sobre DRC em cães e gatos a fim de se obter um melhor direcionamento das ações futuras relativas à prevenção e conscientização sobre a DRC em animais de companhia, que poderão contribuir indiretamente para a redução da mortalidade pela doença e auxiliar na melhoria da qualidade de vida dos animais através de orientação aos tutores.

\section{Material e métodos}

Foi realizada uma pesquisa quantitativa por meio da aplicação de um questionário anônimo estruturado e fechado contendo 17 perguntas, desenvolvido por acadêmicos do curso de medicina veterinária do Centro Universitário FAMETRO, membros da Liga Acadêmica de Medicina Veterinária - LAMVA. O questionário foi elaborado de forma online por meio do Google Forms e disponibilizado em rede social da LAMVA, para captação de participantes voluntários. O link de direcionamento e 
convite para participação na pesquisa também foi enviado pela "lista de transmissão" via aplicativo de mensagens "WhatsApp" para grupos de funcionários de diversas áreas e alunos de variados cursos da instituição. O questionário ficou disponível para preenchimento durante o mês de março de 2019 em decorrência da campanha "Março Amarelo Animal", que teve como objetivo principal conscientizar a população sobre a DRC em animais de companhia.

Todos os participantes preencheram um Termo de Consentimento Livre e Esclarecido - TCLE antes de responderem às perguntas. O questionário foi composto por questões demográficas, bem como questões relacionadas à DRC para investigar o grau de conhecimento dos participantes a respeito da doença, seus sintomas, prevalência e medidas de prevenção, tais como check-up periódico e alimentação adequada.

\section{Resultados e discussão}

A pesquisa contou com 437 participantes, residentes na região de Manaus/AM. Dentre eles, 169 participantes não possuíam nenhum animal de estimação e foram excluídos da análise. Assim, foram analisadas as respostas de 268 participantes. A figura 1 apresenta uma visão geral sobre o perfil destes participantes.

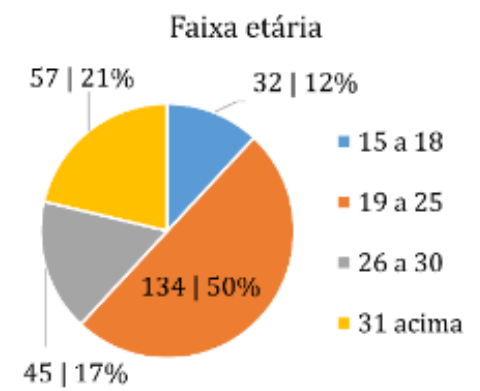

(a)



(b)

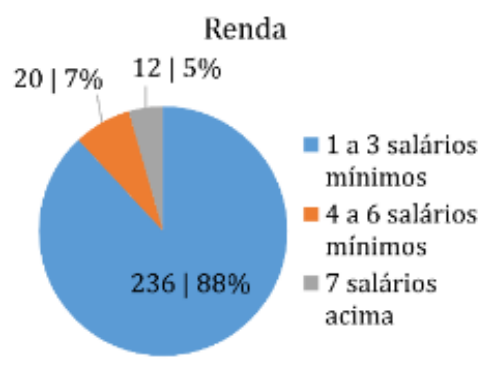

(c)

Figura 1. Informações demográficas dos participantes.

Os participantes eram, em sua maioria, jovens entre 19 e 25 anos (50\%). Quanto à escolaridade, 49\% dos participantes estavam cursando ou finalizaram o ensino médio, enquanto $51 \%$ estavam cursando ou concluíram o ensino superior. Em relação à renda mensal, a grande maioria dos entrevistados possuía renda de 1 a 3 salários-mínimos (88\%).

A figura 2 apresenta o perfil dos respondentes em relação ao número de animais por tutor, frequência de consultas ao médico veterinário e conhecimento sobre a DRC. Em relação à quantidade de animais domésticos (Figura 2a), 81\% relataram possuir pelo menos um cão. Aqueles que informaram possuir ao menos um gato corresponde a $19 \%$ dos participantes.

O resultado da análise da frequência com que tutores com pets os levam ao médico veterinário (Figura 2b) revela que, em sua grande maioria, eles só levam seus animais ao veterinário quando estes estão enfermos (35\%) ou para vacinação (31\%). Uma pequena parte dos tutores (13\%) relatou o hábito de consultas de rotina. Por outro lado, mais de um quinto dos tutores afirmou nunca ter levado seu animal ao médico veterinário $(21 \%)$. Uma análise mais aprofundada mostra a relação entre renda e frequência de consultas regulares ao médico veterinário. Enquanto $42 \%$ dos tutores com renda acima de 7 salários levam seus pets em consultas de rotina, aqueles que recebem entre 1 a 3 salários e de 4 a 6 salários correspondem a somente $11 \%$ e $15 \%$ respectivamente. A baixa assiduidade dos animais às consultas de rotina detectadas nesse trabalho é preocupante e também foi observada por outros autores. Magalhães et al. (2016) por exemplo, apontaram que 56,19\% dos entrevistados em Teresina (PI) não levam seu animal de estimação regularmente ao médico veterinário e $53,57 \%$ o levam somente quando está doente.

Em relação ao conhecimento sobre a DRC (Figura 2c), a maior parte dos participantes relatou nunca ter ouvido falar na doença $(60 \%)$. Pesquisas anteriores sobre o conhecimento da população 
sobre diferentes doenças que acometem animais de companhia apresentaram resultados variados. Sobre leishmaniose visceral canina, por exemplo, Bondan \& Camargo (2015) identificaram que somente 1,4\% dos tutores de pets da cidade de Cotia/SP tinham algum conhecimento sobre leishmaniose visceral canina, muito embora os casos tenham aumentado no estado de São Paulo. Por outro lado, Paulan et al. (2016) relataram que 77\% das famílias de Ilha Solteira/SP declararam conhecer a doença. Sobre a leptospirose, Lee et al. (2020) identificaram que 99,9\% da população (local não especificado) já tinha ouvido falar sobre a doença e $88,2 \%$ conheciam sobre a principal forma de transmissão (por meio de contato com a urina do rato). Sobre cinomose, Lima \& Lallo (2013) apontaram que $80,4 \%$ dos tutores de São Paulo/SP sabiam sobre a doença. Barros \& Albuquerque (2020), por outro lado, apontaram que somente $51,8 \%$ das pessoas entrevistadas em Guarulhos/SP conheciam a doença. Essas grandes variações sobre o conhecimento da população sobre diferentes doenças, inclusive dentro de uma mesma região, como no caso dos estudos conduzidos no estado de São Paulo, reforça a necessidade de se veicular conteúdos informativos e realizar campanhas de conscientização e prevenção a fim de evitar que o ciclo dessas doenças se mantenha na população de animais de companhia.

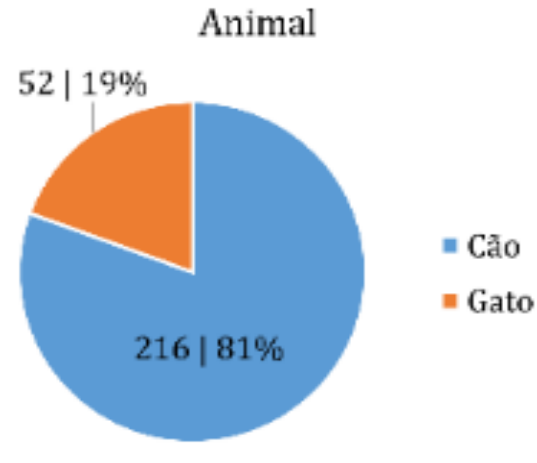

(a)

\section{Conhecimento sobre a DRC}



(c)
Frequência de consultas ao médico veterinário



(b)

Já participou de alguma campanha de prevenção?

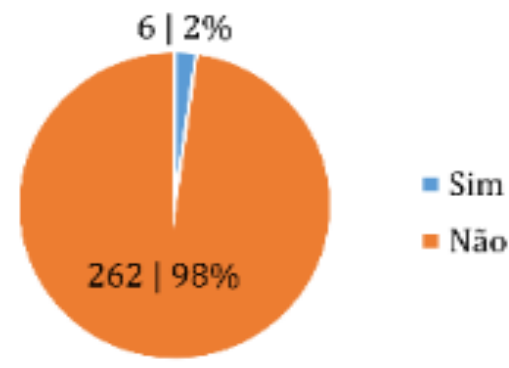

Figura 2. Perfil dos participantes em relação a: (a) Distribuição da quantidade de animais domésticos; (b) Frequência de consultas ao médico veterinário; (c) Conhecimento sobre a DRC; (d) Participação em campanhas de prevenção.

Esta necessidade é reforçada pelos resultados apresentados na Figura 2d, na qual somente $2 \%$ dos participantes já participaram de campanhas de prevenção à DRC. O baixo número de pessoas que têm conhecimento sobre a doença pode estar associado à baixa oferta e participação da sociedade em campanhas de prevenção e conscientização sobre bem-estar animal de pets e as suas enfermidades de maior relevância. Além disso, com a melhoria na qualidade de vida, os pets possuem maior longevidade, fazendo com que doenças que antes não aconteciam com tanta frequência passem a ficar mais rotineiras devido ao aumento da população de meia idade e idosa, reforçando a importância de se realizar 
campanhas desta natureza. É importante ressaltar que campanhas sobre prevenção de doenças renais em animais de companhia ainda não ocorrem com frequência nem em massa; geralmente são realizadas somente uma vez ao ano, com pouca divulgação, o que pode explicar o desconhecimento de grande parte da população em relação à DRC.

$\mathrm{O}$ fato de grande parte das pessoas não levarem seus pets a consultas de rotina prejudica ainda mais o acesso à informação e, associado à falta de conhecimento sobre a DRC pode resultar em situações em que o tutor não percebe os sinais clínicos da doença (principalmente nos estágios iniciais, em que muitas das vezes não são aparentes), levando ao diagnóstico tardio devido à falta de frequência na realização de consultas. Além disso, muitos tutores demoram ou não conseguem associar determinadas alterações como poliúria e polidipsia compensatórias com problemas urinários, o que pode levar ao diagnóstico tardio da doença.

Os resultados sobre a incidência de alterações urinárias apontam que 9\% dos tutores de cães e $10 \%$ dos de gatos relataram que seus animais já foram diagnosticados com algum distúrbio urinário (Figura 3a). Embora poucos casos tenham sido relatados pelos participantes, uma quantidade considerável destes animais é reincidente (Figura 3b), sendo diagnosticados até mesmo três vezes ou mais. Tal resultado pode estar associado, por exemplo, à interrupção no tratamento, levando ao retorno do problema urinário. Vale ressaltar que os tutores não tinham conhecimento para distinguir se as alterações urinárias observadas em seus animais tinham correlação com a DRC. Pesquisas futuras podem incluir a investigação destes casos em específico, buscando identificar os fatores associados às recidivas e identificando quais distúrbios já foram diagnosticados nos animais dos tutores entrevistados.

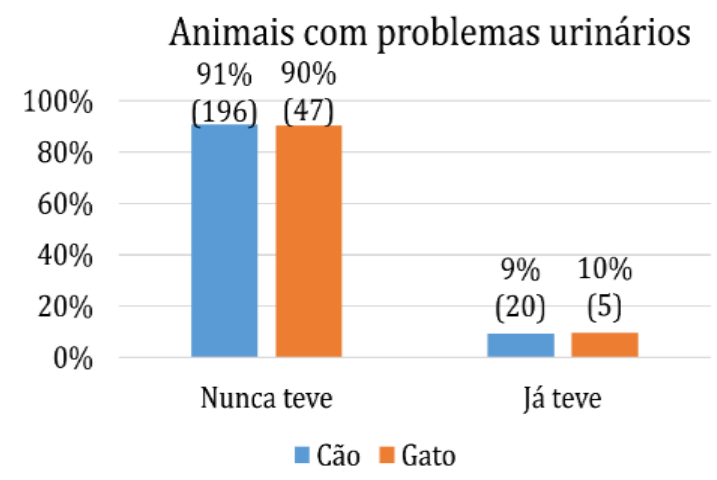

(a)

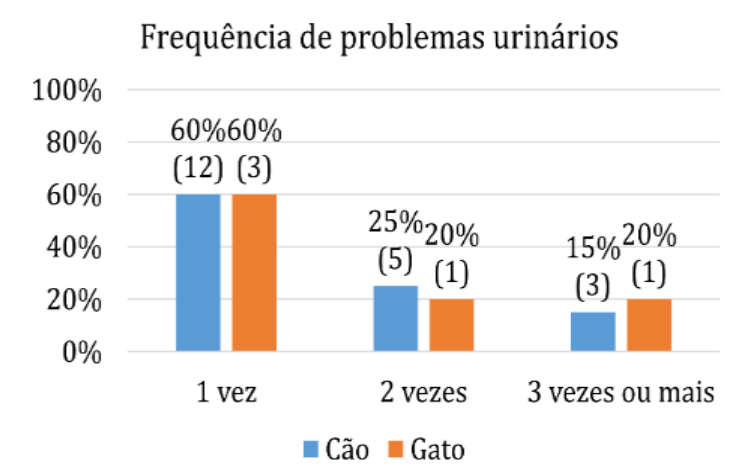

(b)

Figura 3. Quantidade de cães e gatos de acordo com: (a) Frequência de problemas urinários; (b) Frequência de incidência dentre os que foram diagnosticados com problema urinário.

É importante enfatizar que não há muitos trabalhos publicados quanto à percepção dos tutores especificamente para a DRC. Porém trabalhos similares que avaliaram o discernimento de tutores quanto ao bem-estar animal e guarda responsável também observaram a carência de informações dos tutores sobre cuidados básicos médicos veterinários e profiláticos para com os animais de estimação (Pedrassani \& Karvat, 2017).

Com o objetivo de verificar o nível de conhecimento dos participantes sobre a DRC e alguns dos seus sinais clínicos, foram elaboradas as questões apresentadas na Tabela 1. A Figura 4 apresenta os resultados para essas questões.

Como muitos participantes relataram desconhecer a DRC, era esperado que poucas pessoas conhecessem os sintomas e causas associados a esta doença. Contudo, a pesquisa mostra que alguns sinais clínicos e fatores de risco são facilmente associados à DRC. Podemos citar, por exemplo, as questões Q1, Q7 e Q8, que se referem à ingestão de água, alimentação e idade, respectivamente. Por outro lado, sinais clínicos como mau hálito e lesões na cavidade oral (Q3) não são facilmente associados à DRC pelos tutores. Isto pode estar associado ao conhecimento que as pessoas adquirem no dia a dia, 
onde recomenda-se, por exemplo, a ingestão regular de água e o consumo de alimentos saudáveis para manter uma boa saúde, bem como a maior frequência de doenças em pessoas mais idosas. Assim, embora os entrevistados não tenham conhecimento específico sobre a DRC em cães e gatos, esses fatores se tornam de fácil associação com a doença, diferentemente de sinais clínicos mais específicos, como o mau hálito e lesões na cavidade oral.

Tabela 1. Questões relacionadas ao conhecimento sobre os sinais clínicos e causas da DRC.

\begin{tabular}{ll}
\hline \multicolumn{2}{c}{ Descrição } \\
\hline Você sabia que: \\
Q1 & o animal ao ingerir muita ou pouca água, pode ser devido a um problema nos rins? \\
Q2 & o animal ao urinar em grande ou pequena quantidade pode ser devido a um problema renal? \\
Q3 & se o animal apresentar mau hálito ou lesões na cavidade oral, pode estar relacionado a um problema renal? \\
Q4 & existem animais que são predispostos geneticamente a ter Doença Renal? \\
Q5 & os gatos geralmente apresentam problema renal crônico com mais frequência? \\
Q6 & a realização de check-up no seu pet a cada 6 meses pode detectar precocemente uma alteração renal? \\
Q7 & a alimentação pode ser um fator contribuinte para o desenvolvimento de uma Doença Renal Crônica? \\
Q8 & os animais idosos são mais propensos a ter doença renal?
\end{tabular}

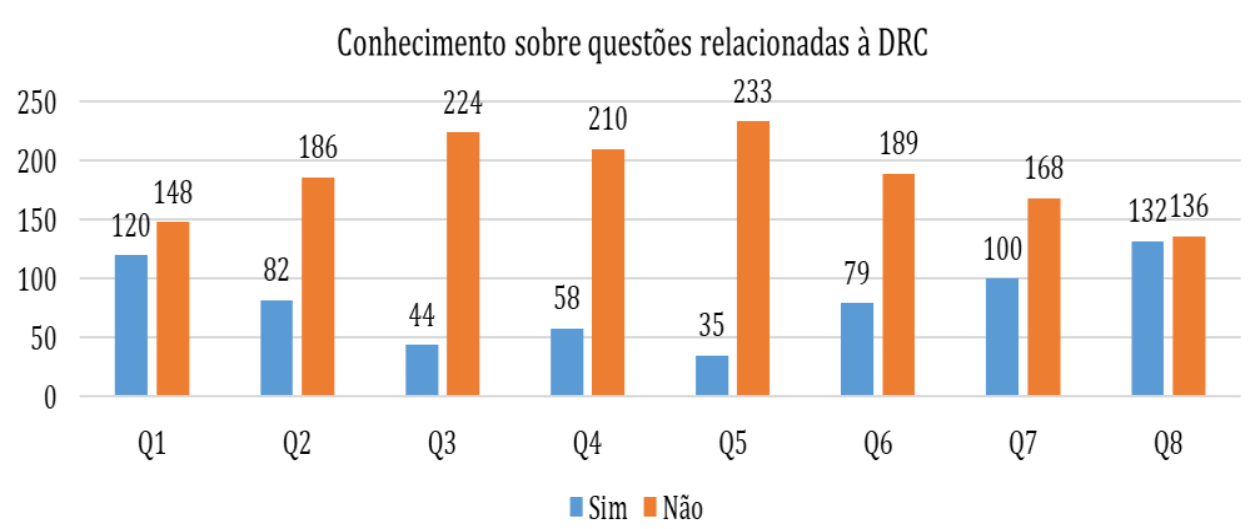

Figura 4. Conhecimento sobre questões relacionadas à DRC.

\section{Conclusão}

O conhecimento dos moradores sobre a Doença Renal Crônica em cães e gatos é limitado. Os entrevistados não são capazes de identificar os sintomas da DRC, sendo o conhecimento insuficiente para prevenção desta grave enfermidade. Notou-se a carência de informações dos tutores sobre cuidados básicos médicos veterinários e profiláticos para com os animais de estimação, o que poderia minimizar riscos de DRC e outras doenças. O aspecto socioeconômico também é um fator importante que está relacionado à frequência de consultas ao médico veterinário, reforçando a necessidade de campanhas de conscientização e prevenção especialmente em locais de baixa renda. Os resultados mostraram também uma taxa considerável de reincidência de alterações urinárias, indicando que os entrevistados talvez estejam negligenciando seus pets por falta de conhecimento e informação. As informações obtidas com o presente estudo reforçam a necessidade de realização de trabalhos educativos constantes sobre bemestar e saúde animal, com ênfase na DRC.

\section{Referências}

Barros, A. A., \& Albuquerque, K. D. (2020). Cinomose canina: Conhecimento popuacional do município de Guarulhos. Revista Saúde-UNG-Ser, 13(2 ESP), 70.

Bondan, E., \& Camargo, T. (2015). Conhecimento sobre leishmaniose visceral canina na população do Município de Cotia (SP), Brasil, e participação dos clínicos veterinários locais na propagação de medidas preventivas. Revista Brasileira de Ciência Veterinária, 22(1), 28-33.

Carvalho, R. L. S., \& Pessanha, L. D. R. (2013). Relação entre famílias, animais de estimação, afetividade e consumo: estudo realizado em bairros do Rio de Janeiro. Revista Sociais e Humanas, 26(3), 622-637. https://doi.org/10.5902/231717586562.

Gerber, F. (2017). Diagnóstico e estadiamento da doença renal crônica em cães. Revista Científica de 
Medicina Veterinária Do UNICEPLAC, 4(2), 46-60.

Lee, B. M. T. C., Pazetti, G., Cattin, I. M., Sordi, M. M., Pilon, V. M., Gonçalves, V. F., \& Santos, E. W. (2020). Avaliação do conhecimento da população sobre a doença leptospirose. PUBVET, 14(12), 1-6. https://doi.org/10.31533/pubvet.v14n12a706.1-6.

Lima, R. S. C., \& Lallo, M. A. (2013). Public survey of knowledge concerning canine distemper and protective measures. Revista Brasileira de Ciência Veterinária, 20(4), 213-215. https://doi.org/10.4322/rbcv.2014.005.

Magalhães, C. S., Lima, W. C., Lima, D. A. S. D., Quessada, A. M., Dornelles, D. E. M., \& Costa Neto, J. M. (2016). Conhecimento de tutores de cães sobre tumor de mama em cadelas. Acta Veterinaria Brasilica, 10(2), 186-189. https://doi.org/10.21708/avb.2016.10.2.5537.

Martínez, P. P., \& Carvalho, M. B. (2010). Participação da excreção renal de cálcio, fósforo, sódio e potássio na homeostase em cães sadios e cães com doença renal crônica. Pesquisa Veterinária Brasileira, 30(10), 868-876. https://doi.org/10.1590/s0100-736x2010001000010.

Paulan, S. C., Silva, D. T., Lins, A. G. S., Lima, F. L., Tenório, M. S., Tasca, K. I., Panosso, A. R., \& Starke-Buzetti, W. A. (2016). O conhecimento sobre leishmaniose visceral: suficiente para controle e prevenção? Revista Ciência Em Extensão, 12(2), 47-60.

Pedrassani, D., \& Karvat, D. C. (2017). Conhecimento sobre bem-estar e guarda responsável de cães e gatos domiciliados e semi-domiciliados. Revista Ciência Em Extensão, 13(4), 55-63.

Polzin, D. J. (2011). Chronic kidney disease in small animals. Veterinary Clinics: Small Animal Practice, 41(1), 15-30.

Queiroz, L. L., \& Fioravanti, M. C. S. (2014). Distúrbios endócrinos na doença renal crônica em cães. Enciclopédia Biosfera, 10(18), 1326-1344.

Togni, M., Curtis, A., Vargas, D. P., Kommers, G. D., Irigoyen, L. F., \& Fighera, R. A. (2018). Causas de morte e razões para eutanásia em gatos na Região Central do Rio Grande do Sul (1964-2013). Pesquisa Veterinária Brasileira, 38(4), 741-750.

Waki, M. F., Ribas Martorelli, C., Erdmann Mosko, P., \& Kogika, M. M. (2010). Classificação em estágios da doença renal crônica em cães e gatos: abordagem clínica, laboratorial e terapêutica. Ciência Rural, 40(10), 1-9.

Histórico do artigo:

Recebido: 3 de março de 2021

Aprovado: 29 de março de 2021.
Licenciamento: Este artigo é publicado na modalidade Acesso Aberto sob a licença Creative Commons Atribuição 4.0 (CC-BY 4.0), a qual permite uso irrestrito, distribuição, reprodução em qualquer meio, desde que o autor e a fonte sejam devidamente creditados. 